\title{
Refreshable Tactile Graphics Applied to Schoolbook Illustrations for Students with Visual Impairment
}

\author{
Grégory Petit, Aude Dufresne Vincent Levesque, Vincent Hayward Nicole Trudeau \\ Department of Communication \\ Haptics Laboratory \\ University of Montréal \\ McGill University \\ Montréal, Canada \\ Montréal, Canada \\ \{gregory.petit, aude.dufresne\} \\ \{vleves, hayward\} \\ @umontreal.ca \\ @cim.mcgill.ca \\ Department of Communication \\ University of Montréal \\ Montréal, Canada \\ trudeaunicole \\ @sympatico.ca
}

\begin{abstract}
This article presents research on making schoolbook illustrations accessible for students with visual impairment. The MaskGen system was developed to interactively transpose illustrations of schoolbooks into tactile graphics. A methodology was designed to transpose the graphics and prepare them to be displayed on the $\mathrm{STReSS}^{2}$, a refreshable tactile device. We experimented different associations of tactile rendering and audio feedbacks to find a model that children with visual impairment could use. We experimented with three scientific graphics (diagram, bar-chart and map) with forty participants: twenty sighted adults, ten adults with visual impairment, and ten children with visual impairment. Results show that the participants with visual impairment liked the tactile graphics and could use them to explore illustrations and answer questions about their content.
\end{abstract}

\section{Categories and Subject Descriptors}

H.5.2 [Information Interfaces and Presentation]: User Interfaces - ergonomics, haptic I/O, input devices and strategies, auditory feedback.

K.3.1 [Computers and Education]: Computer Uses in Education - computer-assisted instruction.

\section{General Terms}

Human Factors, Design, Experimentation.

\section{Keywords}

Students with visual impairment, accessibility, multimodal device, tactile graphics, pedagogy, children with special needs.

Permission to make digital or hard copies of all or part of this work for personal or classroom use is granted without fee provided that copies are not made or distributed for profit or commercial advantage and that copies bear this notice and the full citation on the first page. To copy otherwise, or republish, to post on servers or to redistribute to lists, requires prior specific permission and/or a fee.

ASSETS'08, October 13-15, 2008, Halifax, Nova Scotia, Canada.

Copyright 2008 ACM 978-1-59593-976-0/08/10...\$5.00.

\section{INTRODUCTION}

Schoolbooks take an essential part in the global learning process at school. Even if it is easy to transpose schoolbooks text into Braille, the various pictures, maps and graphics, which illustrate these schoolbooks, are still inaccessible for people with visual impairment. New pedagogical methods rely increasingly on illustrations to convey pedagogical content, partly since they are easier to produce. Schoolbooks have a lot more pictures, often two or three of them on each page, whatever the subject of the book. Moreover, multimedia support is more and more used in classrooms to present some information to the students, while the literacy of people with visual impairment must rely on self-access to paper documents [14].

Even if they can access digital text with screen readers, speech synthesisers and special printers, they still have problem accessing the numerous digital illustrations

Special printers and adapted software now give students with visual impairment access to Braille, but there is still a lot of work to do for the accessibility of illustrations. Most methods to give access to images currently use manual transposition and special papers, such as embossed or thermoform paper [4].

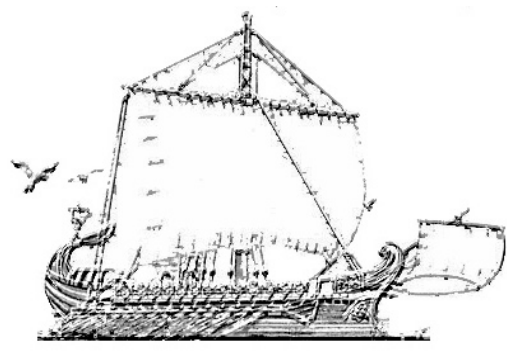

Figure 1. Galley transformed into tactile graphic on special paper

When transposing images for tactile perception it is important to transpose them in order to highlight important elements and hide useless details. For example Figure 1 is difficult to understand by touch since the different parts of the galley are not well marked: it is almost impossible to distinguish the oars and the body of the 
galley. It is generally agreed that adaptation for touch perception is difficult because touch has less resolution than the sight perception, exploration is done linearly and sensibility decreases with time. But it is also hard with paper tactile graphics to transpose a lot of information because all the information remains static (tactile borders, surrounding texts). Moreover, the production of these physical illustrations is very slow, quite expensive and often the images deteriorate with use.

Another solution to give access to images using tactile graphics is to display them on a system which presents refreshable tactile graphics. There are two types of refreshable tactile graphics systems. The first one is a global surface, where all the tactile feedback is already displayed. Thus, the user can explore with his whole hand (or his two hands). For example, the Cat [2] is a small device that was first used to display Braille. But the creators of this device also used it to display simple charts. It is composed of forty Braille cells which each contains sixteen actuators (matrix of $8 \times 2$ ). Each actuator can only be in or out. Thereby, it is hard to have a precise tactile rendering with this device because the actuators only have two positions and they also are quite far from each other. A second example of this kind of devices is the one developed by Shinohara et Al. [11]. This device uses a $20 \times 17 \mathrm{~cm}$ tablet with a matrix of $64 \times 64$ actuators. These actuators are very close to each other and can rise up between $0.1 \mathrm{~mm}$ and $10 \mathrm{~mm}$. This device can give more precise tactile rendering than the Cat. It is easy to have a relief sensation and it is possible to create more elaborate tactile graphics because not only borders are rendered, but also the surface of the shapes.

The second way to digitally produce tactile graphics is to use a tactile cell which can explore a surface. In this case, the tactile rendering is only on the cell and depends on the cell's position on the exploration surface. The VTD (Virtual Tactile Display) [8] is one of these devices. The tactile cell is composed of four Braille cells (the same cells that was used for the Cat system) and can explore a $16.4 \times 15.9 \mathrm{~cm}$ surface. The cell is mounted on a tray which can move using three rails (two horizontal and one vertical). Like the Cat, it is difficult to have a precise tactile rendering because of the Braille cells.

The tactile cell can also be external to the exploration surface. For example, the Tactos device [5] is composed of a Tablet PC and a tactile cell beside the tablet. This tactile cell does not move but the actuators on it move in or out, depending on where the stylus is on the tablet. So, the user uses one hand to hold the stylus and move it upon the table, and he uses his other hand to feel the tactile feedback on the tactile cell.

All these systems can create tactile graphics but they have problem conveying all the necessary information of the original illustration. That's why we used a new multimodal system to create our tactile graphics.

\section{MULTIMODAL DEVICE}

\subsection{Tactile Hardware}

The tactile device used is composed by the STReSS ${ }^{2}$ (Stimulator of Tactile Receptors by Skin Stretch) and the Pantograph (Figure 2). The STReSS [13] can produce tactile feedback by laterally stretching the skin of the finger. This stimulation is performed by the lateral movement of a matrix of $8 \times 8$ piezoelectric bending motors (STReSS ${ }^{2}$ unpublished). Each actuator can deflect toward the left or the right by approximately $0.1 \mathrm{~mm}$. The total tactile feedback area enlarges upon a surface of $12 \times 10.8 \mathrm{~mm}$. A foam cover is placed over the sides of the $\mathrm{STReSS}^{2}$ to insure security and comfort for the user.

The Pantograph [1], developed before the STReSS ${ }^{2}$, is a haptic device which allows two dimensional movements on a surface of $11.3 \times 6 \mathrm{~cm}$. Thereby, you can explore this surface with the STReSS $^{2}$ mounted on it and have a tactile feedback depending where you are on the area.

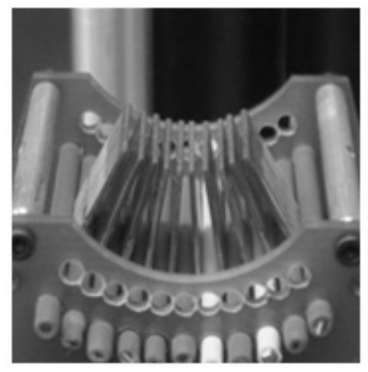

(a)

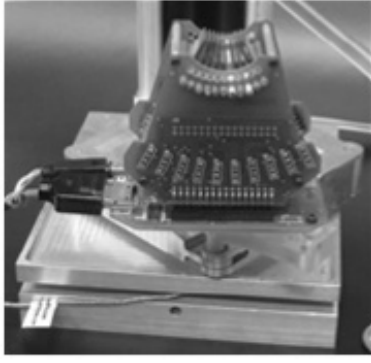

(b)
Figure 2. (a) Actuators on the STReSS 2 and (b) STReSS mounted on the Pantograph.

With this tactile device, it is possible to produce three different kinds of tactile rendering [7] combining the movement on the Pantograph and the deflection of the actuators of the STReSS ${ }^{2}$.

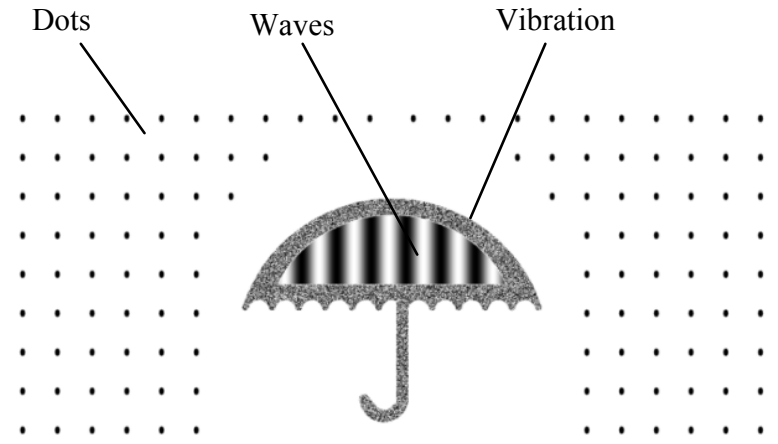

Figure 3. Tactile graphic with the three tactile rendering.

First, as you can observe in Figure 3, you can have a dot sensation like when people with visual impairment are reading Braille. Then, it is also possible to feel a vibration. This sensation is important because you can feel it even if you don't move the device. The last tactile rendering is the wave sensation. In this 
case, you can feel an undulation underneath your finger when you move the device. These waves can have different orientations and spacing. The essential point is that it is possible to combine all these types of tactile rendering in order to obtain a full tactile transcription of an illustration.

\subsection{The STReSS ${ }^{2}$ Software}

The STReSS ${ }^{2}$ tactile device is controlled by software programmed in $\mathrm{C}++$ and running on a personal computer with Linux as Operating System. This software is using the Xenomai real-time framework in order to have a tactile feedback produced at $1 \mathrm{kHz}$. To display a tactile graphics, the illustration must have been decomposed into several parts called "masks". Each part corresponds to a subdivision of the illustration with which we associate a tactile feedback and/or an audio feedback. Thereby, it is possible to associate different feedbacks to different parts of the illustration and to combine them in order to produce the complete tactile graphics. The description of the different parts is written in an XML file which is read by the STReSS ${ }^{2}$ system. This XML file is used to set the different settings of the tactile rendering like the frequency of the tactile feedback or the orientation of the undulations when the wave sensation is selected. This file is also used to set the audio feedback.

In this XML file, there is a primitive for each mask, beginning by the type of tactile rendering like $"<$ vibrogen $>$. With each primitives, the different properties are defined for each mask, like the orientation angle in the undulation rendering (<angle units $=$ "degree" $>45<$ /angle $>$ ) or the image file for the shape ( $<$ imgfile $>80000$.png $<$ /imgfile $>$ ).

\section{TACTILE ADAPTATION}

In order to transpose an illustration into tactile graphics that could be read with the STReSS${ }^{2}$ system, we used the methodology developed by Trudeau [12]. The objectives of her work were to establish an optimal method to make tactile adaptation of illustrations in schoolbooks, for students with visual impairment. She wanted to analyse the illustrations in a structural, systematic and synthetic way, taking a realistic account of how they could be explored by students with visual impairment in a learning context, using tactile perception. Thereby, she created what she calls "cards" where the relevant properties of an illustration are described. She made three different types of analysis card: analysis card for a book, for a page and for an illustration. We mainly used the analysis cards for illustrations in our project.

These cards were used to more correctly produce tactile graphics. In most cases, it is the graphic designer who decides how to transform an illustration into a tactile graphic. So the adaptation can be different depending on the designer's feelings or understanding of what is relevant. We wanted to more clearly define how the different components of an illustration should be transposed into tactile parameters. Thus, we used the methodology developed by Trudeau to produce our tactile graphics in a more systematic way, for example: keeping only significant elements, increasing edges, erasing patterns, completing and highlighting parts even when they are not very apparent to the eye. So we used the cards she had made to create images, which have been used in our research.

In the parameters of an illustration card, there is a detailed description of the illustration. This description is essential to correctly identify the pedagogical purpose of each illustration. Actually, this purpose is different for each illustration. A map and bar-chart don't usually have the same purpose. A bar-chart is used to show the proportion or an evolution of something during a period, whereas a map is used to present spatial organization. Moreover, for each illustration, there is an "Essential" section in its analysis card, which is useful to not forget any important information during its transformation into tactile graphics, separating useful and useless information.

In her work, Trudeau also provides general ergonomic recommendations to follow in order to facilitate the adaptation. For example, it is good to briefly comment on the tactile graphics before the user starts to explore it. Moreover, if the original illustration is composed of different level of information, it is better to create one tactile graphics for each level of information and to allow the user to switch between the different tactile graphics. These ones should be produced at the same scale in order to keep the same proportions in all the tactile graphics composing the whole illustration.

Previous research has shown that the vibration rendering is very powerful and easy to perceive but tends to cause perceptual fatigue if it is too extensively used [7]. It was therefore decided to use the vibration tactile rendering to accentuate the essential information in our tactile graphics. It is important to the user to know when he is leaving an area of the illustration to enter another one. So, we decided to attribute the vibration rendering to the different borders of the tactile graphic. Thereby, the user would be able to easily follow the outline of each area composing an illustration.

\section{TACTILE GRAPHICS PRODUCTION}

All the original illustrations we used were extracted from the general history schoolbook "Enjeux et Découvertes", Tome 1 [10]. This book is for students who are between 13 and 14 years old.

As previously explained, we cannot use an illustration like it is in order to produce a tactile graphic. It has to be adapted. At the moment, it is difficult to automatically do this adaptation although some researchers are starting to do it [6]. So we developed the MaskGen system to automate some steps of the adaptation and facilitate the edition of the final transposition into a multimodal description, used by the $\mathrm{STreSS}^{2}$ system to allow visually handicapped users to explore the image with tactile and audio feedback.

First, the original illustration is simplified with an editing tool like Photoshop or The Gimp to remove unnecessary elements such as text. To integrate the images with the $\mathrm{STreSS}^{2}$ system, we have to produce a tactile graphic where all the borders will be in black and 
the several parts inside these borders in white. Those borders have to be thickened and less smooth because, if they are too thin and too elaborate, it will be impossible for the users to correctly identify them. Various filters in Photoshop can be used to transform the image: modify color for greyscale, simplify rendering, extract, smooth and enlarge edges. For example, this is the original illustration we used in our experiment (Figure 4).

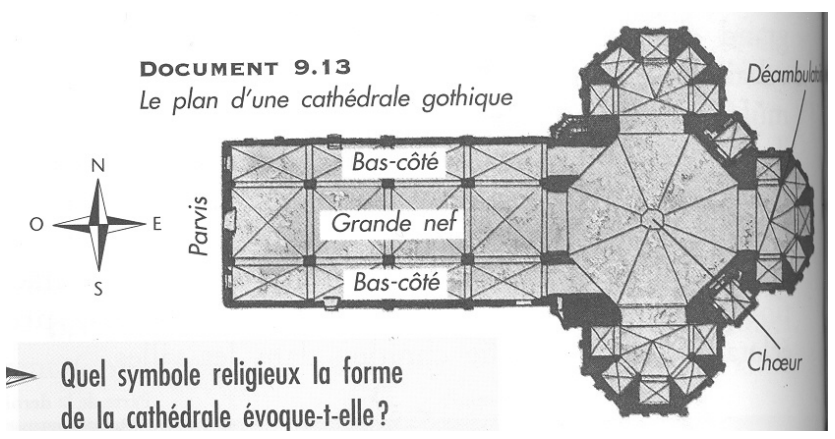

Figure 4. Original illustration of the layout of a gothic cathedral.

The Figure 5 shows the simplified illustration obtained using Photoshop.

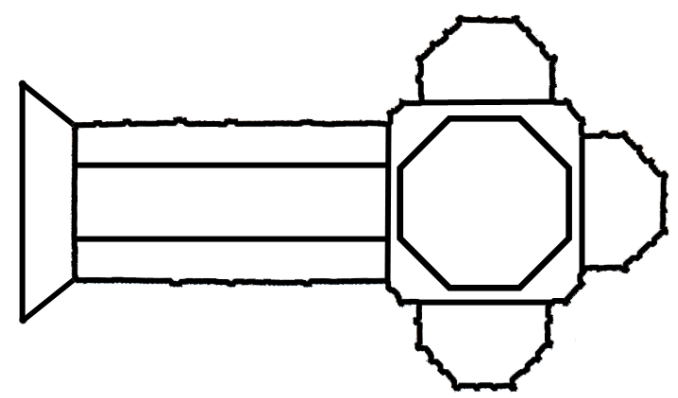

Figure 5. Simplified illustration of the cathedral.

Once images have been manually simplified, they can then be opened with the MaskGen system, which will automatically extract all the different parts of this kind of simplified illustration. When all the parts have been extracted as different masks, it is possible to use MaskGen to ascribe to each one a specific tactile feedback, an audio feedback and to adjust the settings of the tactile feedback. The audio feedback can be produced by speech synthesis (like a robot's voice) reading the description text,in French or English, by voice recording with a microphone or by using an existing audio file. It is also possible to merge several parts if we want them to have the same feedback or to eliminate them if they are irrelevant. When the different parts of the illustration and their feedbacks are ready, MaskGen automatically produce the XML file which will be used by the STReSS ${ }^{2}$ system. The tactile graphics is then ready to be explored by the user. This preparation takes between 5 to 15 minutes for the preliminary work on Photoshop, depending on the complexity of the illustration. Then, MaskGen conclude the transposition (maximum 5 minutes)
We adapted three tactile graphics coming from original illustrations of the general history schoolbook. The first one was the plan of a gothic cathedral (see Figure 4). The second illustration (Figure 6) was a bar-chart showing the population of Athens sorted by social belonging, five centuries before Jesus Christ. And the third illustration (Figure 7) was a world map showing the different civilizations which first appeared on earth.

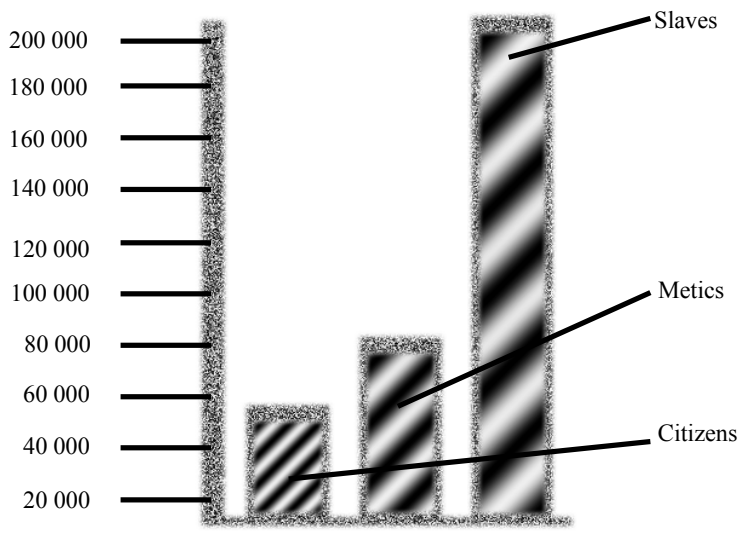

Figure 6. Distribution of the Athens' population

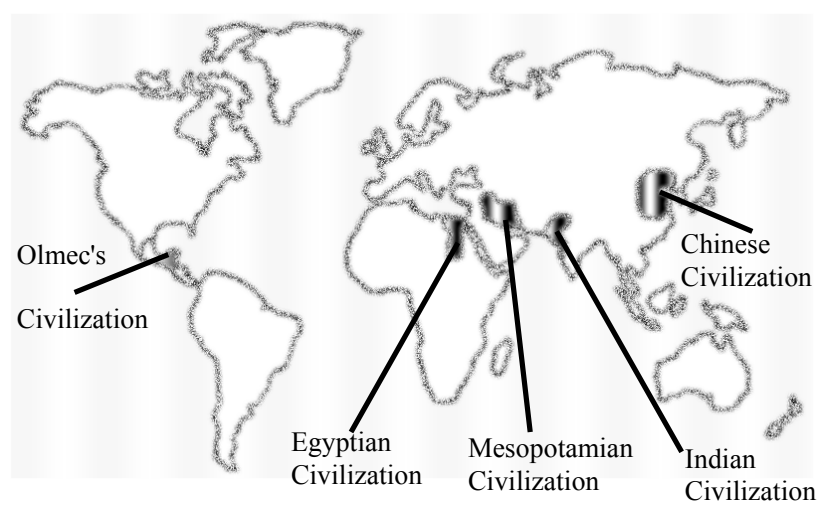

Figure 7. The first civilizations.

In this map, the users needed to find the different civilizations that were there and to localize them inside the continents. So as suggested by Trudeau [12], we prepared two different levels of information for this tactile graphic, one for the civilizations and one for the continents. Each level of information was defined as a separate illustration in MaskGen with a set of different shapes and their feedback, so the user could explore them. These two levels of information were made with the same scale, so the user could superimpose the information by moving from one level to the other. The user could switch between the two tactile graphics using a keyboard button. When he used it, he was exactly at the same place on the second tactile graphics as he was on the first one. This gradual exploration, with different level of details, is certainly an important aspect of the system, with no equivalent in previous ones. 


\section{EXPERIMENTATION}

The main purpose of this experimentation was to check if the tactile graphics we produced could serve the pedagogical purpose as the original illustrations in the schoolbook. We wanted to test different methods of tactile rendering and audio feedback.

The experiment first involved twenty sighted persons with a blindfold over their eyes; the second one was made with twenty participants with visual impairment: ten adults and ten children (average of 15.4 years old). We involved sighted people because it is difficult to find participants with visual impairment, especially children. We also wanted to check if sighted users could be as effective as users with visual impairment at evaluating the system. Actually, it is a cliché to say that people with visual impairment have a better tactile sensation than sighted people. We wanted to know if this observation was right using our device.

We used vibration rendering for the different outlines of the tactile graphics, but we also wanted to check if it was useful to add tactile rendering inside the different areas composing the shapes. So, we split each group of twenty persons in two sub-groups. The first one (VU) had vibration rendering for the outlines and undulation rendering in the inside areas. The second one (V) only had vibration rendering on the outlines with no tactile rendering inside the different areas.

For this experimentation, we did not use dots rendering at this stage. In all cases, audio feedback was used so the participant was informed of the nature of the area in which he was entering. For the audio feedback, we used voice recording for the two tactile graphics and the speech synthesis for the third one (cathedral). First, each participant was given a few minutes to explore simple shapes with the multimodal device. They explored the different kinds of tactile renderings and got accustomed with the control of the device since the exploration area was quite small and graphic were downsized to fit into it. Then, for each of the three illustrations, the experimenter read to the participant a brief description of the content of the image. He also gave an example of question which could be asked at the end of the exploration of the illustration. Then, the participant freely explored the illustration for five minutes.

After that, the experimenter asked the participant to answer some questions about the illustration, like: "In which continent was the Olmec civilization?", "How many citizens were there according to the bar-chart?". There were thirteen questions in all the illustrations, which were rated from 0 to 10 . The users could still explore with the STReSS ${ }^{2}$ to find the answer if they wanted to. The purpose of these questions was to check if the participant had correctly located the different areas and correctly understood the pedagogical content of the illustration. Some of these questions were also on the book with the original illustrations. We used these ones because it was the original idea from the author to check if the students have correctly understood the illustration. Participants were told that it was not a problem if they could not answer a question because the touch sensation is quite different for each person. Thereby, we obtained a score for each illustration, which is the mean percentage they obtained in their answers on a specific illustration. The time they took to explore and answer questions was also recorded.

When the exploration and questioning on each illustration was over, the experimenter proceeded to the final questionnaire. In this questionnaire, we asked the participants what he would like to improve in our system, if there was something he would like to remove, if he thought he understood the tactile graphics, which illustration was the easier to understand... The experimenter read each question to the participant with or without visual impairment, so the conditions were the same for both.

We also recorded the experimentation with a camera in order to analyse the different exploration strategies used by the participants. Thus, we were able to observe how they interacted with the device and which methods they used to better understand the illustrations.

\section{RESULTS}

The main result of this experiment is certainly that the participants with visual impairment, especially children, really liked the device and were able to use it to understand images. They obtained good scores on the content questions (see Table 1).

Table 1. Mean scores and time spent for each illustration of users with (S) or without visual impairment (VI).

\begin{tabular}{|c|c|c|c|c|c|}
\hline & Group & $\mathrm{Nb}$ & $\begin{array}{c}\text { Mean. } \\
\%\end{array}$ & $\begin{array}{c}\text { Expl. } \\
\text { Time }\end{array}$ & $\begin{array}{c}\text { Quest. } \\
\text { Time }\end{array}$ \\
\hline \multirow{2}{*}{ Cathedral } & $\mathrm{S}$ & 20 & 69,70 & 222 & 100 \\
\cline { 2 - 6 } & $\mathrm{VI}$ & 20 & 74,80 & 184 & 138 \\
\hline $\begin{array}{c}\text { Attic's } \\
\text { population }\end{array}$ & $\mathrm{S}$ & 20 & 85,88 & 207 & 71 \\
\cline { 2 - 6 } & $\mathrm{VI}$ & 20 & 89,38 & 231 & 101 \\
\hline $\begin{array}{c}\text { Civilizations } \\
\text { map }\end{array}$ & $\mathrm{S}$ & 20 & 87,00 & 257 & 108 \\
\cline { 2 - 6 } & $\mathrm{VI}$ & 20 & 77,75 & 286 & 190 \\
\hline \multirow{2}{*}{ Total } & $\mathrm{S}$ & 20 & 80,00 & 686 & 279 \\
\cline { 2 - 6 } & $\mathrm{VI}$ & 20 & 80,19 & 701 & 429 \\
\hline
\end{tabular}

This means that the pedagogical purpose of the tactile graphics has been reached, since they correctly answered the different questions using the multimodal device.

We did not find significant differences on the scores that the participants obtained for the illustrations, depending on the following independent variants: blindfolded sighted people / people with visual impairment; group VU / group V; adults with visual impairment / children with visual impairment. This observation could be related with the major individual variance we noticed on the different populations who participated on our experimentation. We found a standard deviation of 12.8 about the age of our participants. Moreover, some people with visual impairment had this impairment since they were born and some had it later, which is why we have this major individual variance. 


\subsection{Differences between Sighted Participants and Participants with Visual Impairment}

We found no significant difference between the blindfolded sighted and the participants with visual impairment for the total time and the exploration time spent on the illustration. Our experimentation cannot really confirm that people with visual impairment have a better tactile sensation than sighted people because we also used an audio feedback and the sight users told us it was really helpful for them to have this audio feedback (even more helpful than the tactile rendering). But it seems that participants with visual impairment spent more time answering our questions $(\mathrm{F}=3.98 ; \mathrm{p}=0.05)$.

Moreover, it appears that even if the sighted participants had previous knowledge on the different kinds of illustrations, they were no better at understanding them using the multimodal device. Looking for each question (with a t-test), there is one question, which showed some difference. About the civilizations map $(p=0.049)$ the sighted had a better score, which was: "Which civilization is more to the East on the map". It was obvious, for someone who had seen a world-map before, and knew the localisation of the continents, that the Chinese civilisation was the right answer, compared to the Indian or the Egyptian ones. This observation suggests that sighted persons were able to have a better virtual representation of the illustration because they had already seen a similar illustration before, contrary to people with visual impairment. But we can wonder if this observation would remain true if the two both populations had explored illustrations showing objects of everyday life or something that they don't both know.

The same is true when we look at the time spent for each illustration (Figure 8). Moreover, the global time taken by the participants with visual impairment answering the questions is longer $(F=3.3982 ; p=0.54)$, especially for the civilizations map $(\mathrm{F}=3.58 ; \mathrm{p}=0.044)$.

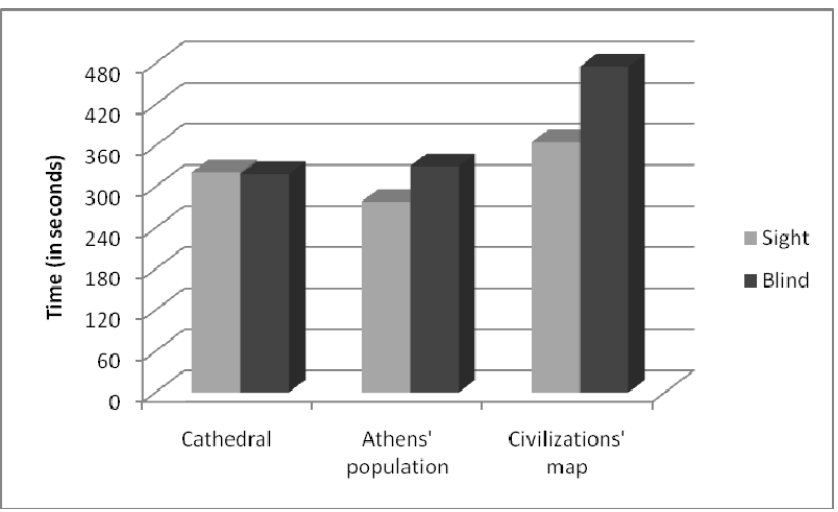

Figure 8. Graph showing the time spent for each illustration, depending of the handicap of the participant

We also found another significant difference in the questionnaire we gave to the users. Participants with visual impairment reported that they better understood the tactile graphics than the sighted participants with blindfold on their eyes. We asked them if the tactile graphics were easy to understand. On a scale from 1 to 5 , the mean for the participants with visual impairment was 4 whereas the mean for the sighted one was only $2.9(\mathrm{p}=0.001)$. So, even if, the two populations obtained the same scores, the participants with visual impairment reported it was easier to understand with the tactile graphics. With more time to practice with tactile graphics, they might become quite efficient and find it easier.

\subsection{Differences between Adults with Visual Impairment and Children with Visual Impairment \\ We can also find some significant differences between adults with} visual impairment and children with visual impairment. The scores are similar but there is a significant difference in the time they took to perform the experiment. The children did all the experiment faster. They took five minutes less than the adults with visual impairment $(\mathrm{p}=0.011)$ to perform all the experiment and, as you can see in the Figure 9, they did it faster for all the three illustrations. Even if it shows that children with visual impairment always complete the tasks a little faster than adults with visual impairment, only the plan of the Cathedral is significant $(\mathrm{p}=$ $0.012)$.

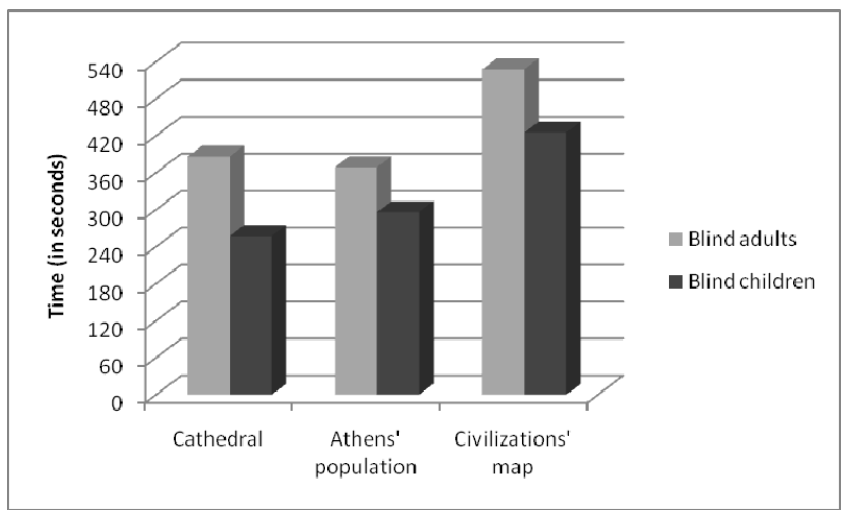

Figure 9. Graph showing the time spent for each illustration, depending of the age of the participant

\subsection{Differences Depending on the Tactile Rendering in the Shapes}

About the group with vibration rendering for the outlines and undulation rendering in the inside areas (VU), and group with only vibration rendering and without tactile rendering inside the different areas (V), we did not find any significant differences between them, even if, participants from group VU seemed to do the experiment a little faster .

In the questions, we found two significant differences. The first one was to guess what the global form of the gothic cathedral was. It seems that the participants from group VU answered faster to this question $(p=0.014)$. This suggests that you can recognise the global form of an object faster when you have a texture 
(undulation rendering) inside the different areas that compose the object. This observation may be linked to another observation. In the bar-chart which showed the population of Athens sorted by their social belonging, we asked the participants to tell us which social belonging was the least represented on the bar-chart. Once again, participants from the group VU answered faster to this question than participants from group $\mathrm{V}$ though globally they obtained the same score. That suggests that they perceived faster the size of each bar.

\subsection{Audio Feedback}

We wanted also to know which audio feedback, between speech synthesis and voice recording, was the best in our multimodal system. It seems that the participants slightly understood the voice recording better $(\mathrm{p}=0.046)$. The mean for the speech synthesis was $79 \%$ and the one for the voice recording was $87 \%$. Many users said this should be set by a preference, but results mean that in some conditions, where voice recording is not practical, that textual speech synthesis would be acceptable, as when we want to easily translate the illustrations description.Audio feedback was found essential, though participants would have liked to have more control over it. They did not like the fact that it was repeated when they were exploring borders. Moreover, in the map, we tried to use a sound as a metaphor for water, which was found very irritating.

\subsection{Video analysis}

We observed which elements were the most difficult to explore thanks to the tactile sensation in the three illustrations, and which exploration strategies were the most used by the participants. To do this, we used the MORAE software [15] which helped us to code the videos by adding some tags to them. We only coded those from the participants with visual impairment.

Among the different exploration strategies, the "following outlines" strategy matches with the fact of following outlines of the illustration thanks to the tactile sensation. The "saccade" strategy consists on going from a specific zone to another specific one in fits and starts. A "missed zone" is when the user goes over a specific zone without noticing it. The "systematic scan" strategy is used when the user explores the illustration in a well-defined way, like from the top to the bottom or circumnavigating it. The "random scan" strategy happens when the user explores the illustration without taking into account the tactile and audio feedbacks. The "reach a goal" strategy is used when the user directly goes to a zone he has already identified. Finally, the "starting point" strategy happens when the user comes back at his starting point, on the bottom of the left.

On the Figure 10, we can notice that the participants did not really follow the outlines on the civilizations map whereas it was the most used strategy for the two other illustrations. This can be explained by the fact that this map has more details than the two other illustrations. That's why the outlines must be more difficult to follow. This explanation is also conceivable to justify the high number of "missed zone" on the civilizations map because the civilizations are very small, in comparison of the size the world map. Moreover, the "random scan" strategy was often used on the map in order to randomly locate the small civilizations.

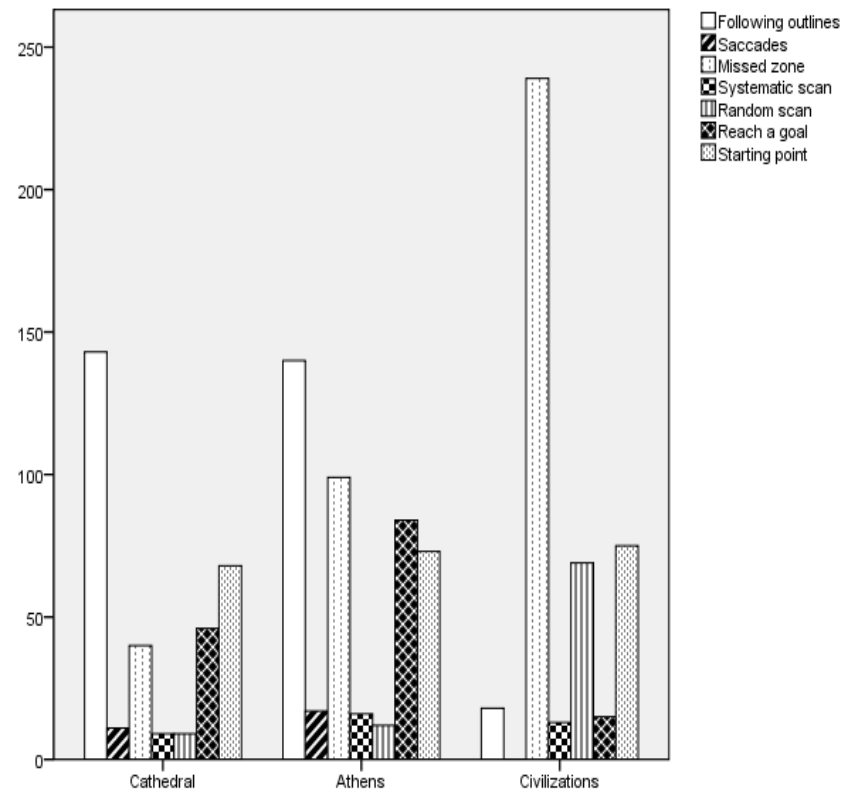

Figure 10. Exploration strategies for each illustration

As we said, the "following outlines" strategy was the most used one on the illustration of the cathedral and the bar-chart. This shows that the participants mainly used the tactile feedback to understand these two illustrations. We also observed that the participants more often came back at their original point when they were answering to the questions. Finally, they more often followed the outlines during the exploration phase (215 tags) than the questions phase ( 85 tags).

\section{CONCLUSION}

To conclude this paper, we have produced a multimodal device to adapt tactile graphics for children with visual impairment. These tactile graphics were reproducing illustrations in a history schoolbook. We wanted to know if tactile graphic could convey the pedagogical purpose as their original version. It appears that this main purpose has been accomplished since our participants succeeded well in answering the various questions and they also reported that they easily understood the illustrations. Moreover, the children really liked using our device. They asked many questions about how it was working, and if we will soon go back to their school to do other tests. One of them was particularly excited and was always repeating "It's so cool!". They also appear to do the experimentation faster than adults with visual impairment.

About the tactile rendering, we first thought that adding textures (with undulation rendering) inside the several parts which compose an illustration would really help the user to better understand the tactile graphics. But we only found one significant 
difference between the two groups: adding textures inside the shapes helped the participant to have a better idea of the global shape of the illustration. Adding textures maybe is not as useful as we expected, but we would need more experimentation to see how it could help, in other contexts, such as when maps have bigger zones, when elements of a legend are repeated, etc. But the advantage we found is nevertheless important. Moreover, the participants told us in their comments when they filled our questionnaire, that using two different tactile graphics with the same scale was very useful to show several levels of information.

Thanks to the analysis of the videos, we observed that the more elaborate the illustration is, the more difficult it is to use the tactile feedback to understand the illustration. That's why the previous work which is done to adapt the original illustration is very important in order to take off the irrelevant information.

At the moment, the multimodal device still has some limitations. We cannot currently create very complex tactile graphics because of the size of the exploration area. Future research will include improving the system to transpose more complex illustrations, exploring the level of information, vector graphics, a bigger exploration space and maybe a zoom feature. We would also like to automate the process that adapts an original illustration into a simplified graphic. For now, we used contour extraction, borders enlargement, and filling shapes and texts in Photoshop, these could be made automatic, or other existing programs could be used.

Moreover, we would like to use our system to make accessible a whole digital document, like web-pages, with text and picture. In order to access a digital document, users with visual impairment only have access to the text and they use speech synthesis that move from the left top to the right bottom. With the STReSS ${ }^{2}$, the user could explore the structure of the entire document and select the part he wants to access, like in the PCAccess system [3];[9], where the Pantograph or a mouse is used to explore the screen. This could be the next steps for this new technology, which has a great potential according to some of our users.

\section{ACKNOWLEGMENTS}

This research was supported by a FQRNT grant. We would like to thank all the subjects who participate to our experimentation, Pierre Ferland and Marie-Chantal Wanet from the "Institut Nazareth et Louis Braille", and the school for visually handicapped students Jacques Ouellette for giving us the opportunity to test our system with some their students.

\section{REFERENCES}

[1] Campion, G., Wang, Q., and Hayward, V. The pantograph MK-II: a haptic instrument. In Proc. IROS 2005, pages 193198, 2005.

[2] Ebina, T., Igi, S., Miyake, T. and Takahashi, H.. Graph Access System for the Visually Impaired. In Proc. of the Third Asian Pacific Computer and Human Interaction. Kangawa, Japon, 1998.
[3] Dufresne, A., Martial, O. and Ramstein, C. (1995). Multimodal User Interface System for blind and "visually occupied" users: Ergonomic evaluation of the Haptic and Auditive Dimensions. In Interact'95 (pp. 163-168). Lillehamer.

[4] Edman. P. K. Tactile Graphics. AFBPress, New York, 1992.

[5] Gapenne, O., Rovira, K., Ammar, A. Ali and Lenay, C. Tactos: Special computer interface for the reading and writing of 2D forms in blind people, In Universal Access in HCI, Inclusive Design in the Information Society, pages 1270-1274, 2003.

[6] Ladner, R. E., Ivory, M. Y., Rao, R., Burgstahler, S., Comden, D. Hahn, S., Renzelmann, M., Krisnandi, S., Ramasamy, M. , Slabosky, B., Martin, A. Lacenski, A., Olsen, S. and Groce. D.. Automating tactile graphics translation. In Proceedings of the 7th international ACM SIGACCESS conference on Computers and accessibility (Assets '05), pages 150-157, New York, NY, USA, 2005. ACM Press.

[7] Levesque, V. and Hayward, V. Tactile Graphics Rendering Using Three Laterotactile Drawing Primitives. In Proceedings of the 16th Symposium on Haptic Interfaces For Virtual Environment And Teleoperator Systems. Reno, NV, USA, March 2008.

[8] Maucher, T., Schemmel, J. and Meier, K. The Heidelberg Tactile Vision Substitution System. In International Conference on Computers Helping People with Special Needs (ICCHP2000). Karlsruhe, Germany, July 2000.

[9] Ramstein, C., Dufresne, A., Martial, O., Carignan, M. Chasse, P., \& Mabilleau, P. (1996). Touching and Hearing GUI's: Design Issues for the PC-Access System. Paper presented at the International Conference on Assistive Technologies, Vancouver, Canada.

[10] Roby, J. and Paradis, C. Enjeux et Découvertes, Tome 1 (manuel de l'élève), Éditions HRW - Group Éducalivres inc., Second trimestre, 1995.

[11] Shinohara, M., Shimizu, Y. and Mochizuki, A. ThreeDimensional Tactile Display for the Blind. In IEEE Treansactions on Rehabilitation Engineering, Vol. 6, $N^{\circ} 3$, pages 249-256, September 1998.

[12] Trudeau, N. «Analyse visuelle du livre, de la page et de l'image du manuel de l'élève Enjeux et découvertes.» Final report of quinquinal research project, Montréal, 2000-2005.

[13] Wang, Q. and Hayward, V. Compact, portable, modular, high-performance, distributed tactile transducer device based on lateral skin deformation. In Proc. HAPTICS'06, pages6772, 2006.

[14] http://www.cnib.ca/fr/apropos/publications/accessibilite/droit -de-lire/Default.aspx.

[15] http://www.techsmith.com/morae.asp 